\title{
The Role of Transvaginal Ultrasound Guided Embryo Transfer to Improve Pregnancy Rate in Obese Patients Undergoing Intracytoplasmic Sperm Injection
}

\author{
Sarah Mohamed Hassan (D) \\ Wafaa Ramadan (D) \\ Mohammed Elsharkawy (D) \\ Yomna Ali Bayoumi \\ Department of Obstetrics and \\ Gynecology, Cairo University, Cairo, \\ Egypt
}

\begin{abstract}
Objective: To evaluate the effectiveness of transvaginal ultrasound (TVUS) versus the transabdominal ultrasound (TAUS) guided embryo transfer (ET) in obese patients undergoing Intracytoplasmic sperm injection (ICSI).

Design: A prospective randomized controlled trial.

Setting: University hospital.

Patient(s): Eight hundred participants with BMI $\geq 30$ received the long agonist protocol. They were randomized into 2 equal groups of 400 patients. Each group had either TAUS guided ET or TVUS guided ET.
\end{abstract}

Main Outcome Measure(s): Our primary outcomes were clinical pregnancy rate, degree of patient pain assessed by visual analogue scale (VAS). The secondary outcomes were live birth rate, implantation rate, miscarriage and chemical pregnancy rates, duration of ET.

Result(s): Our results showed a significant higher chemical and clinical pregnancy rates in the TVUS group in comparison to TAUS group $(45.3 \%$ vs. $38.3 \%, \mathrm{p}=0.045)$ and $(37.8 \% \mathrm{vs}$. $30.8 \%, p=0.044$ ) respectively. There was tendency for increased live birth rates in the TVUS group however it did not reach statistical significance $(50.2 \%$ vs. $44.8 \%)$. The VAS of the pain was significantly less in TVUS group in comparison to the TAUS group $(2.1 \pm 0.7 \mathrm{vs}$. $4.5 \pm 1.3$ ), also abdominal discomfort was significantly less in TVUS group in comparison to the TAUS group $(13 \%$ vs. $58 \%) \mathrm{p}=$ value 0.0001 .

Conclusion(s): This study showed superior role of TVUS guided ET over TAUS regarding less pain and better pregnancy and live birth rates in obese participants.

Clinical Trial Registration Number: Clinical trial. Gov identifier NCT03473028.

Keywords: IVF/ICSI, obesity, embryo transfer, ET

\section{Introduction}

With increasing incidence of delayed conception and infertility, the ultimate need to improve the results of assisted reproduction techniques (ART) became the major concern of many researchers worldwide. ${ }^{1}$ Many ART cycle failures despite good oocyte and embryos quality were attributed to suboptimal embryo transfer (ET) technique, ${ }^{2}$ and so attempts to improve ET techniques might increase ART success rates by increasing implantation rates. ${ }^{3}$

Historically, ET technique was performed in a blinded clinical touch technique. With advances in ET procedure, the use of transabdominal ultrasound (TAUS) guided
Correspondence: Sarah Mohamed Hassan Tel +20100373367l

Email saramohamed7880@yahoo.com 
ET has been introduced. Sticklers et al in 1985 was the first one to describe the benefit of the use of US for ET. ${ }^{4}$ Since then there is good and consistent evidence of the benefit of ultrasound guidance during the process of ET. ${ }^{5}$ More than 20 randomized clinical trials comparing the clinical touch technique with the use of TAUS were studied in three large Meta-analysis $^{6-8}$ and a Cochrane review. ${ }^{9}$ The use of transvaginal ultrasound (TVUS) guided ET was first described in $1990 .^{10}$ In contrast to TAUS, the use of TVUS guided ET does not require full bladder so avoiding pain and patient discomfort, allows better visualization of catheter tip during transfer and provides better resolution of uterocervical angle leading to more favorable pregnancy outcome. ${ }^{10-12}$

Our objective is to compare TVUS versus TAUS ET in obese patients undergoing ICSI as regards the clinical pregnancy rate, the patient discomfort and pain, the live birth rate, the implantation rate, the miscarriage rate, chemical pregnancy rate and ET duration.

\section{Materials and Methods}

This is a prospective randomized controlled trial conducted at Kasr Al Ainy IVF unit in Cairo, Egypt, during the period April 2018, to February 2021.

It included participants who were candidates for ICSI and had the following inclusion criteria: (1) age between 25-40 years with BMI $\geq 30$; (2) good ovarian reserve (Antral follicular count from 8-15, FSH $<12 \mathrm{mIU} \backslash \mathrm{mL}$, AMH 1.5 to $3.5 \mathrm{ng} / \mathrm{mL}$ ); and (3) unexplained infertility more than 3 years. Our exclusion criteria included: (1) all couples with severe male factor of infertility; (2) any uterine factor of infertility detected by ultrasonography and hysteroscopy; (3) patients with ovarian endometrioma or polycystic ovary syndrome; (4) obesity caused by endocrine dysfunction eg Thyroid or adrenal disorder; and (5) patients with previous multiple ICSI failure ( $>5$ trials).

All patients in our study underwent the following: History taking, general examination including vital signs, BMI calculation, abdominal and pelvic examination. Baseline 2D TVUS to assess AFC using "Mindray DP5" set, Day 3 serum sample for FSH, LH, E2, prolactin, $\mathrm{TSH}$ Serum and anti-Mullerian hormone (AMH).

The Ethics Committee of Kasr Al Ainy approved the study and it was registered on clinical trial.gov (NCT03473028).

\section{ICSI Used Protocol}

We used the long down regulation protocol which entails daily subcutaneous injection of triptorelin (Decapeptyl;
Ferring, Saint-Prex, Switzerland) at $0.1 \mathrm{mg}$ started on day 21 of the cycle preceding the stimulation cycle, and the dose was reduced to $0.05 \mathrm{mg} /$ day on the day of the following menstruation till the day of human chorionic gonadotropin (hCG) triggering. After confirming pituitary down regulation criteria (thin endometrium $[<5 \mathrm{~mm}]$, serum estradiol $[<50$ pmol/Land no visible ovarian cysts]), ovarian stimulation by HMG (Merional 75 IU; Menotropins FSH 75 IU; Menotropins LH 75 IU) (IBSA, InstitutBiochimique SA, Lugano, Switzerland) with a starting dose ranging from 150IU-300IU according to ovarian reserve and patient age. Repeated ultrasound scans were performed using a 5.0-9.0 $\mathrm{MHz}$ multifrequency transvaginal probe (Mindray DP-5). When the mature follicles reach 18-20 mm HCG administration (choriomon ${ }^{\circledR}$, IBSA 10000 IU) was done. TVUSguided oocyte retrieval is performed 34-35 hours following HCG injection. Progesterone vaginal tablets (prontogest ${ }^{\circledR}$, IBSA) are administered $400 \mathrm{mg}$ twice daily as luteal support from the day of the oocyte retrieval. All fresh ETs were done day 3 using either TAUS or TVUS guidance technique, according to the study allocation. All cases were subjected to mock ET on the day of oocyte retrieval to predict the ease of the procedure. This is a routine practice in our unit. Difficult cases that needed use of tenaculum were included in our study, however very difficult cases that needed the introduction of uterine sound were excluded. No analgesia or sedation were given to the patient on the day of ET. All ETs were performed by the same operator with high level of experience using the Edwards-Wallace catheter (Classic Embryo Replacement Catheter; Smiths Medical).

The allocation to the TAUS group or TVUS was done on the day of ET through randomization based on sealed numbered envelopes opened by the resident in charge before the procedure; the allocation ratio was $1: 1$. Written informed consent was signed by all participants who accepted to participate.

\section{ET Technique}

In the TVUS group, the technique was described Karavani et $\mathrm{al}^{1}$ and by Kojima et al. ${ }^{11}$ Participants were instructed to empty their bladder. The cervix was exposed by Cusco speculum and cleansed gently by a sterile gauze pad, the outer sheath of the ET catheter was introduced through the external OS and then the speculum was removed. The physician inserts the TVUS probe covered with sterile condom into the vagina. He holds the TVUS probe with the left hand and manipulates it till obtaining a sagittal view of the uterine cavity and cervix. The nurse fixes the 
outer sheath till the physician introduces the soft inner catheter loaded with the embryos into the outer sheath. The soft inner catheter was introduced through the internal OS into the uterine cavity under ultrasonographic guidance. If there is any difficulty, the physician withdraw the inner sheath and the outer sheath was introduced through the internal OS but not into uterine cavity. Difficult cases were defined as those needed to grasp the cervix by a tenaculum or presence of blood in the catheter during the mock transfer. The same technique was followed for such difficult cases without removing the speculum and applying simple traction on the tenaculum.

The catheter was advanced under TVUS guidance to a distance $1-2 \mathrm{~cm}$ from tip of catheter to the uterine fundus. The catheter and the ultrasound probe were removed after confirming the appearance of two echogenic spots caused by the two loaded air bubbles by TVUS. The catheter was checked by the attending embryologist for any retained embryos. Cases with retained embryos were retransferred by the same technique. At the end of the procedure, patients rest in bed for 20 minutes.

In the TAUS group, patients were instructed to drink water to fill their urinary bladder. The nurse held the probe in the suprapubic location showing uterine sagittal axis with the full urinary bladder. Exposure of cervix by Cusco speculum and ET catheter were introduced in the same technique described above in the TVUS group.

\section{Evaluation of Pain by VAS}

Evaluation of patient discomfort and degree of pain during transfer were assessed by Questionnaire Design based on a visual analogue scale (VAS) graded from $0-10$.The patients recorded the VAS scores by marking along a $10 \mathrm{~cm}$ line $0=$ no abdominal discomfort; $10=$ worst abdominal discomfort imaginable. VAS was assessed at the end of ET procedure.

\section{Study Outcomes}

Our primary outcomes were

1. clinical pregnancy rate it was diagnosed by the presence of a positive heart beat by TVUS in a the gestational sac 5 weeks after positive B HCG

2. degree of patient pain and assessed by VAS

The secondary outcomes were:
1. Live birth rate, which was defined as the number of achieved live births after 28 weeks' gestation.

2. Implantation rate which was calculated as the ratio of the number of gestational sacs to the number of embryos transferred

3. Miscarriage and ongoing pregnancy rates. Early miscarriage was defined as clinical pregnancy loss before 12 weeks' gestation. Ongoing pregnancy was defined as pregnancy continuing beyond 12 weeks' gestation.

4. Chemical pregnancy was diagnosed by serum B-HCG level >100 IU/L 14 days after ET.

5-Duration of ET was calculated from time the ET catheter was given to the transferring operator till the time it was handed back to the transferring embryologist. If any embryos were retained, the total duration was calculated from the time of the start of technique till the time required for final successful ET.

\section{Sample Size Calculation}

It was done based on comparing 2 proportions from independent samples using Chi test, the $\alpha$-error level was fixed at 0.05 and the power was set at $80 \%$. As previously published by Laure et al $2017,{ }^{13}$ using conventional TAUS achieved clinical pregnancy rate of $30 \%$ while TVUS is expected to achieve $10 \%$ more in pregnancy rate. Accordingly, the optimum sample size should be 356 cases in each arm. Sample size calculation was done using PS Power and Sample Size Calculations software, version 3.0.11 for MS Windows (William D. Dupont and Walton D. Vanderbilt, USA).

\section{Statistical Analysis}

Data were described in terms of mean \pm standard deviation ( $\pm \mathrm{SD}$ ), range and median, or percentages, frequencies (number of cases) when appropriate. Comparison of numerical variables was done using Student's $t$-test for independent samples. For comparing categorical data, Chi-square $\left(\chi^{2}\right)$ test was performed. Exact test was used instead when the expected frequency is less than 5. $p$ values less than 0.05 was considered statistically significant. All statistical calculations were done using computer program IBM SPSS (Statistical Package for the Social Science; IBM Corp, Armonk, NY, USA) release 22 for Microsoft Window.

\section{Results}

The final number randomized into either TAUS or TVUS guided ET were 800 participants, 400 in each group. 
Table I Baseline Characteristics of the Study Population

\begin{tabular}{|c|c|c|c|}
\hline & Transabdominal US $(n=400)$ & Transvaginal US $(n=400)$ & $P$ value \\
\hline Age & $31.58 \pm 3.6$ & $31.7 \pm 3.6$ & NS \\
\hline BMI & $35.2 \pm 2.39$ & $35.37 \pm 2.4$ & NS \\
\hline D3 FSH & $7.67 \pm 1.2$ & $7.83 \pm 1.3$ & NS \\
\hline D3 LH & $6.3 \pm 1.09$ & $6.23 \pm 0.7$ & NS \\
\hline D3 AMH & $2.16 \pm 0.5$ & $2.12 \pm 0.49$ & NS \\
\hline AFC & $15.21 \pm 2.7$ & $14.9 \pm 2.57$ & NS \\
\hline \multirow[t]{4}{*}{ Previous failure } & $0(28 \%)$ & $0(36 \%)$ & \multirow[t]{4}{*}{ NS } \\
\hline & I (48\%) & I (38\%) & \\
\hline & $2(21 \%)$ & $2(19 \%)$ & \\
\hline & $3(3 \%)$ & $3(7 \%)$ & \\
\hline Endo Thickness & $11.79 \pm 1.27$ & $11.67 \pm 1.42$ & NS \\
\hline No oocyte & $13.02 \pm 2.36$ & $12.76 \pm 2.27$ & NS \\
\hline MII & $9.44 \pm 1.87$ & $9.29 \pm 2.08$ & NS \\
\hline MI & $0-5(3)$ & $0-6(4)$ & \\
\hline E2 pick up & $2569 \pm 495.23$ & $2530 \pm 455.4$ & NS \\
\hline No oocytes fert & $8.42 \pm 1.85$ & $8.17 \pm 1.89$ & NS \\
\hline \multirow[t]{2}{*}{ No embryos trans } & $2(84 \%)$ & $2(82 \%)$ & \multirow[t]{2}{*}{ NS } \\
\hline & $3(16 \%)$ & $3(18 \%)$ & \\
\hline Grade A & $5.2 \pm 1.69$ & $5.01 \pm 1.5$ & NS \\
\hline Grade B & $2.06 \pm 0.85$ & $2.07 \pm 0.6$ & NS \\
\hline Retained embryos & $9 / 400(2.3 \%)$ & $5 / 400(1.3 \%)$ & NS \\
\hline
\end{tabular}

Notes: A $\mathrm{P}$ value $<0.05$ is considered statistically significant. Data were described in terms of mean \pm standard deviation ( \pm SD), range and median, or percentages. Comparison of numerical variables was done using Student's $t$-test for independent samples. For comparing categorical data, Chi-square $\left(\chi^{2}\right)$ test was performed.

No statistically significant differences were found in the Baseline characteristics between the two groups (Table 1).

Our results showed significant higher chemical and clinical pregnancy rates in the TVUS group in comparison to the TAUS group $(45.3 \%$ vs. $38.3 \% \mathrm{p}=0.0045)$ and $(37.8 \%$ vs. $30.8 \% \mathrm{p}=0.0044)$ respectively. We had no cases of ectopic pregnancy in both groups. The VAS of the pain during the ET was significantly less in TVUS group in comparison to the TAUS group $(2.1$ \pm 0.7 vs. $4.5 \pm 1.3 \mathrm{p}=0.0001$ ), also the abdominal discomfort was significantly less in TVUS group in comparison to the TAUS group $(13 \%$ vs. $58 \% \mathrm{p}=0.0001)$ (Table 2). Regarding the live birth rate, it was higher in transvaginal group in comparison to Transabdominal group $(50.2 \%$ vs. $44.8 \%)$. However, the difference was not statistically significant. There is no statistically significant difference between both groups regarding the implantation rate, and the incidence of abortions. The duration of ET in seconds was longer in TAUS group in comparison to the TVUS group ( $201.8 \pm 42.4$ vs. 197.19 $\pm 27.3)$ but this difference was not statistically significant (Table 2).

The incidence of cases who experienced difficult ET was higher in TAUS group in comparison to the TVUS group $(7.8 \%$ vs. 5) The chemical pregnancy rate, the clinical pregnancy rate, the live birth rate per clinical pregnancy and the live birth rate per difficult ET were all higher in TVUS vs. TAUS group. However the differences were not statistically significant (Table 3 ). 
Table 2 Outcomes Among the Study Women

\begin{tabular}{|l|l|l|l|}
\hline & Transabdominal US (n = 400) & Transvaginal US (n = 400) & P value \\
\hline VAS during transfer & $4.5 \pm 1.3$ & $2.1 \pm 0.7$ & $0.000 I^{*}$ \\
\hline ET Duration & $201.8 \pm 42.4$ & $197.19 \pm 27.3$ & NS \\
\hline Clinical pregnancy & $123 / 400(30.8 \%)$ & $151 / 400(37.8 \%)$ & $0.044^{*}$ \\
\hline Live birth rate & $179 / 400(44.8 \%)$ & $201 / 400(50.2 \%)$ & NS \\
\hline Live birth delivery rate & $103 / 400(25.8 \%)$ & $126 / 400(31.5 \%)$ & NS \\
\hline Miscarriage & $20 / 123(16.3 \%)$ & $26 / 151(17.2 \%)$ & NS \\
\hline Implantation rate & $231 / 864(27 \%)$ & $234 / 872(26.8 \%)$ & NS \\
\hline Chemical pregnancy & $152 / 400(38.3 \%)$ & $181 / 400(45.3 \%)$ & $0.045 *$ \\
\hline Abdominal discomfort & $232 / 400(58 \%)$ & $52 / 400(13 \%)$ & $0.000 I^{*}$ \\
\hline Difficult ET & $31 / 400(7.8 \%)$ & $20 / 400(5 \%)$ & NS \\
\hline
\end{tabular}

Notes: *A P value $<0.05$ is considered statistically significant.Data were described in terms of mean \pm standard deviation ( \pm SD), or percentages. Comparison of numerical variables was done using Student's $t$-test for independent samples. For comparing categorical data, Chi-square $\left(\chi^{2}\right)$ test was performed.

Table 3 Analysis in Difficult ET

\begin{tabular}{|l|l|l|l|}
\hline & Transabdominal US (n= 3I) & Transvaginal US (n = 20) & P value \\
\hline Chemical pregnancy & $5 / 31(16.1 \%)$ & $7(35 \%)$ & NS \\
\hline Clinical pregnancy & $5 / 31(16.1 \%)$ & $7(35 \%)$ & NS \\
\hline Ongoing pregnancy & $2 / 5(40 \%)$ & $4 / 7(57 \%)$ & NS \\
\hline Live-birth rates (per clinical preg) & $2 / 5(40 \%)$ & $4 / 7(57 \%)$ & NS \\
\hline Live-birth rates (per difficult ET) & $2 / 31(6.4 \%)$ & $4 / 20(20 \%)$ & NS \\
\hline
\end{tabular}

Notes: All values presented as percentage. A $P$ value $<0.05$ is considered statistically significant. For comparing categorical data, Chi-square $\left(\chi^{2}\right)$ test was performed.

\section{Discussion}

ET step remains a crucial factor which affects the success of IVF cycles. ${ }^{14-16}$

The most novel meta-analysis comparing TVUS versus TAUS ET was studied by Cozzolino et al $2018^{17}$ stated that only 4 studies comparing this topic were done up to date, 3 of which were randomized controlled studies ${ }^{1,2,18}$ and 1 was an observational one. ${ }^{13}$

According to our knowledge, our current study is the first randomized controlled study in literature comparing the effectiveness of transvaginal versus TAUS guided ET in obese participants undergoing IVF as regards to ease of procedure, abdominal discomfort and clinical pregnancy.

Our study showed significant higher chemical and clinical pregnancy rates in TVUS group compared to TAUS group. These results were consistent with Larue et al study ${ }^{13}$ which showed marked increase in pregnancy rate with TVUS ( $38 \%$ vs. $30 \% ; \mathrm{p}=0.0004)$. Our hypothesis is that TVUS provides better visualization of uterine cavity, catheter tip and ET location as showed in one Metaanalysis that embryo placement at $20 \mathrm{~mm}$ provides higher pregnancy rates than those placed at $10 \mathrm{~mm} .{ }^{19}$ A previous study on 120 participants found statistically significant difference in visualization of uterus and ET location favoring TVUS group over TAUS group despite no statistical difference in the clinical pregnancy rate. ${ }^{1}$

However, our result does not go with the results by Karavani et al, ${ }^{1}$ who showed no difference in clinical pregnancy rates between the two groups, this might be explained by lower number of patients included in that study which was 60 in each arm. The same results were analyzed by another study ${ }^{18}$ who showed no significant difference in clinical pregnancy rates with transvaginal group compared with transabdominal group $(50.9 \%$ vs. 
49.4\%), our hypothesis to that is that study was conducted on donor oocyte participants and with different ovarian stimulation protocol, with different operators performing the transfer step and also because our study was conducted only on obese $(\mathrm{BMI} \geq 30)$ where TVUS provides better visualization of transferred embryos and subsequently better chance of implantation.

One of the major advantages of TVUS ET is the need of single operator participants even though it needs training and experience as it implicates handling the transvaginal probe and the ET catheter accurately at the same time. As regards the TAUS ET, it requires the presence of an extra well trained personnel, additionally TVUS has better resolution due to its proximity to the target organs allowing proper visualization of catheter tip especially in cases with obesity and marked uterine retroversion. ${ }^{18}$ Interestingly, the previous two randomized studies comparing TVUS vs. TAUS showed the similar results regarding implantation rate. ${ }^{1,18}$ TVUS has superior role in cases with difficult transfer because it can easily analyze the cause of difficult transfers as endocervical crypts, marked anteverted uterus, cervical canal tortuosity. In our study, there was non-significant increase in the number of difficult transfers in transabdominal group compared to the transvaginal group $(7.8 \%$ vs. $5 \%$ respectively). The clinical pregnancy rates and live birth rates in difficult transfers were higher in the transvaginal group but this difference did not reach statistical significance $(35 \%$ vs. $16.1 \%$ and $20 \%$ vs. $6.4 \%$ respectively). However, the numbers were not adequately high enough to assess this. Also, the percentage of retained embryos was higher in the TAUS group (2.3\%) compared to the TVUS group (1.3\%) but with no statistical significance. Similar result was showed by Bodri et $\mathrm{al}^{18}$ who showed $3.6 \%$ retained embryos in TAS and $1.8 \%$ in TVS group. This could be attributed to more proper positioning of catheter tip in TVUS group and so more precise embryo deposition in uterine cavity with minimal mucosal trauma. ${ }^{20}$

Another important advantage of TVUS is requiring a fully emptied urinary bladder in contrast to the TAUS which necessitates proper bladder distension which is both time consuming and also causes abdominal discomfort and uterine cramps leading to increased patient anxiety. Theoretically, this could lead to embryos expulsion thus reducing the implantation and pregnancy rates. ${ }^{21}$

As regards to abdominal discomfort and uterine cramps, there was extremely high statistical significance between the two groups in our study where $58 \%$ of participants had abdominal discomfort in TAUS group while only $13 \%$ had discomfort in TVS group (p 0.0001), this was consistent with a recent study by Karavani et al (2017) where participants reported less pain and discomfort before, during, and after the procedure in TVUS group compared to TAUS group. Same results reported by a previous study showing $63 \%$ abdominal discomfort in TAUS group related to bladder distension. ${ }^{18}$

Based on VAS questionnaire graded from 0-10, participants reported less pain during the transfer in TVS group compared to TAS group (2.1 vs. 4.5), this difference was highly statistically significant. The strength of this study was related to its randomized nature and that all transfers where done by single operator so eliminating any bias. Moreover, as previously mentioned, this is the first study in literature comparing TVUS versus TAUS ET in obese participants.

\section{Conclusion}

This study showed prominent superior role of TVUSguided ET over TAUS in obese participants. Our main limitation was that we had to exclude cases with very difficult transfer who needed sedation or anesthesia. We hope for more studies in the future addressing this issue with more number of participants.

\section{Data Sharing Statement}

Availability of data and material: the authors intend to share individual deidentified participant data, regarding the demographic data and the results, if required anytime, you can contact Yomna Ali Bayoumi, ${ }^{1}$ M.D.

\section{Ethics}

The study was conducted in accordance with the Declaration of Helsinki and Ethics approval from obstetrics and gynecology scientific committee.

\section{Consent to Participate}

All patients enrolled in the study consented to participate.

\section{Synopsis}

A Randomized controlled trial showed superior role of TVUS-guided ET over TAUS as regards better pregnancy and live birth rates in obese patients undergoing ICSI.

\section{Acknowledgments}

The authors thank all of the female participants and the staff of the Cairo University IVF Center for their help and support. 


\section{Funding}

There is no funding to report.

\section{Disclosure}

All authors declare no conflict of interest.

\section{References}

1. Karavani G, Ben-Meir A, Shufaro Y, Hyman JH. Ariel Revel Transvaginal ultrasound to guide embryo transfer: a randomized controlled trial. FertilSteril. 2017;107:1159-1165.

2. Porat N, Boehnlein LM, Schouweiler CM, Kang J, Lindheim SR. Interim analysis of a randomized clinical trial comparing abdominal versus transvaginal ultrasound-guided embryo transfer. $J$ Obstet Gynaecol Res. 2010;36:384-392. doi:10.1111/j.1447-0756.20 09.01148.x

3. Mansour RT, Aboulghar MA. Optimizing the embryo transfer technique. Hum Reprod. 2002;17:1149-1153. doi:10.1093/humrep/ 17.5.1149

4. Strickler RC, Christianson C, Crane JP, et al. Ultrasound guidance for human embryo transfer. Fertil Steril. 1985;43:54-61. doi:10.1016/ S0015-0282(16)48317-2

5. Mains L, Van Voorhis BJ. Optimizing the technique of embryo transfer. Fertil Steril. 2010;94(3):785-790. doi:10.1016/j. fertnstert.2010.03.030

6. Buckett WM. A meta-analysis of ultrasound-guided versus clinical touch embryo transfer. Fertil Steril. 2003;80:1037-1041. doi:10.1016/S0015-0282(03)01015-X

7. Sallam HN, Sadek SS. Ultrasound-guided embryo transfer: a meta-analysis of randomized controlled trials. Fertil Steril. 2003;80:1042-1046. doi:10.1016/S0015-0282(03)01009-4

8. Abou-Setta AM, Mansour RT, Al-Inany HG, Aboulghar MM, Aboulghar MA, Serour GI. Among women undergoing embryo transfer, is the probability of pregnancy and live birth improved with ultrasound guidance over clinical touch alone? A systemic review and meta-analysis of prospective randomized trials. Fertil Steril. 2007;88:333-341. doi:10.1016/j.fertnstert.2006.11.161

9. Brown J, Buckingham K, Abou-Setta AM, Buckett W. Ultrasound versus'clinical touch' for catheter guidance during embryo transfer in women. Cochrane Database Syst Rev. 2010;CD006107.

10. Hurley VA, Osborn JC, Leoni MA, Leeton J. Ultrasound-guided embryo transfer: a controlled trial. Fertil Steril. 1991;55:559-562. doi:10.1016/S0015-0282(16)54185-5
11. Kojima K, Nomiyama M, Kumamoto T, Matsumoto Y, Iwasaka T. Transvaginal ultrasound-guided embryo transfer improves pregnancy and implantation rates after IVF. Hum Reprod. 2001;16:2578-2582. doi:10.1093/humrep/16.12.2578

12. Anderson RE, Nugent NL, Gregg AT, Nunn SL, Behr BR. Transvaginal ultrasound-guided embryo transfer improves outcome in patients with previous failed in vitro fertilization cycles. Fertil Steril. 2002;77:769-775. doi:10.1016/S0015-0282(01)03279-4

13. Larue L, Keromnes G, Massari A, et al. Transvaginal ultrasound-guided embryo transfer in IVF. J Gynecol Obstet Hum Reprod. 2017;46:411-416L. doi:10.1016/j.jogoh.2017.02.015

14. Edwards RG. Clinical approaches to increasing uterine receptivity during human implantation. Hum Reprod. 1995;10(Suppl. 2):60-66. doi:10.1093/humrep/10.suppl_2.60

15. Hoozemans DA, Schats R, Lambalk CB, Homburg R, Hompes PG. Human embryo implantation: current knowledge and clinical implications in assisted reproductive technology. Reprod Biomed. 2004;9:692-715. doi:10.1016/S1472-6483(10)61781-6

16. Irani M, Reichman D, Robles A, et al. Morphologic grading of euploid blastocysts influences implantation and ongoing pregnancy rates. Fertil Steril. 2017;107:664-670. doi:10.1016/j.fertn stert.2016.11.012

17. Cozzolino M, Vitagliano A, Di Giovanni MV, et al. Ultrasoundguided embryo transfer: summary of the evidence and new perspectives. A systematic review and meta-analysis. Reprod Biomed. 2018;3 (6):524-542. doi:10.1016/j.rbmo.2018.01.015

18. Bodri D, Colodron M, Garcia D, Obradors A, Vernaeve V, Coll O. Transvaginal versus transabdominal ultrasound guidance for embryo transfer in donor oocyte recipients: a randomized clinical trial. FertilSteril. 2011;95:2263-2268.

19. Abou-Setta AM. What is the best site for embryo deposition? A systematic review and meta-analysis using direct and adjusted indirect comparisons. Reprod Biomed. 2007;14:611-619. doi:10.1016/S1472-6483(10)61054-1

20. Cenksoy PO, Ficicioglu C, Yesiladali M, Akcin OA, Kaspar C. The importance of the length of uterine cavity, the position of the tip of the inner catheter and the distance between the fundal endometrial surface and the air bubbles as determinants of the pregnancy rate in IVF cycles. Eur J Obstet Gynecol Reprod Biol. 2014;172:46-50. doi:10.1016/j.ejogrb.2013.09.023

21. Lesny P, Killick SR, Tetlow RI, Robinson J, Maquiness SD. Embryo transfer: can we learn anything new from the observation of junctional zone contractions? Hum Reprod. 1998;13:1540-1546. doi:10.1093/humrep/13.6.1540

\section{Publish your work in this journal}

The International Journal of Women's Health is an international, peerreviewed open-access journal publishing original research, reports, editorials, reviews and commentaries on all aspects of women's healthcare including gynecology, obstetrics, and breast cancer. The manuscript management system is completely online and includes a very quick and fair peer-review system, which is all easy to use. Visit http://www.dovepress.com/testimonials.php to read real quotes from published authors. 